\title{
INCLINAÇÃO DE CABEÇA DURANTE A MASTIGAÇÃO HABITUAL NAS DEFORMIDADES DENTOFACIAIS CLASSE II E III
}

\author{
Head inclination during habitual chewing in the presence \\ of class II and III dentofacial deformities
}

\author{
Marcela Ralin de Carvalho Deda ${ }^{(1)}$, Melissa Nara de Carvalho Picinato-Pirola (2), \\ Francisco Veríssimo de Mello-Filho ${ }^{(3)}$, Luciana Vitaliano Voi Trawitzki ${ }^{(4)}$
}

\begin{abstract}
RESUMO
Objetivo: este estudo investiga a diferença entre grupos com deformidade dentofacial (padrão classe II e classe III), em relação à preferência mastigatória referida e inclinação de cabeça durante a mastigação, assim como predomínio intra-grupo de cada um desses aspectos, comparativamente a um grupo controle. Método: participaram deste estudo, voluntariamente, 25 pacientes (entre 18 e 35 anos). Doze pacientes com diagnóstico de deformidade dentofacial classe II e 13 pacientes com o diagnóstico de classe III esquelética e 10 voluntários, com equivalência em sexo e idade ao grupo de deformidade, formando o grupo controle. Os voluntários da pesquisa foram questionados sobre a existência de um lado de preferência mastigatória. Em seguida foram filmados durante a mastigação habitual de um biscoito maizena e através das imagens captadas, a postura de cabeça pôde ser analisada. Resultados: não houve diferença entre os grupos em relação à preferência mastigatória referida, entretanto os grupos com deformidades dentofaciais apresentaram predomínio significativo de preferência mastigatória referida. Os grupos com deformidade dentofacial apresentaram maior predomínio de inclinação de cabeça durante a mastigação quando comparados ao grupo controle. Conclusão: indivíduos com deformidade dentofacial podem apresentar uma preferência mastigatória e alteração de postura de cabeça durante a mastigação.
\end{abstract}

DESCRITORES: Má Oclusão; Postura; Cabeça; Mastigação

\section{INTRODUÇÃO}

A fisiologia da mastigação é caracterizada por ciclos unilaterais com alternância periódica do

(1) Fisioterapeuta; Pós-graduanda em Ciências Médicas pela Faculdade de Medicina de Ribeirão Preto da Universidade de São Paulo, FMRP/USP, Ribeirão Preto, São Paulo, Brasil; Mestre em Ciências Médicas pela Faculdade de Medicina de Ribeirão Preto da Universidade de São Paulo.

(2) Fonoaudióloga; Pós-graduanda em Ciências Médicas pela Faculdade de Medicina de Ribeirão Preto da Universidade de São Paulo, FMRP/USP, Ribeirão Preto, São Paulo, Brasil; Mestre em Ciências Médicas pela Faculdade de Medicina de Ribeirão Preto da Universidade de São Paulo.

(3) Médico; Professor da Faculdade de Medicina de Ribeirão Preto da Universidade de São Paulo, FMRP/USP, Ribeirão Preto, São Paulo, Brasil; Livre-docente.

(4) Fonoaudióloga; Professora, Faculdade de Medicina de Ribeirão Preto da Universidade de São Paulo, FMRP/USP, Ribeirão Preto, São Paulo, Brasil; Doutora em Biociência aplicada à Clínica Médica pela Faculdade de Medicina de Ribeirão Preto da Universidade de São Paulo.

Conflito de interesses: inexistente alimento entre os dois lados da arcada dentária1. Quando a mastigação ocorre predominantemente em um lado específico da cavidade oral, durante o ciclo mastigatório, considera-se lado de preferência mastigatória ${ }^{2}$ ou predomínio mastigatório. Estudos têm mostrado que indivíduos sadios podem apresentar um predomínio unilateral nessa função ${ }^{1,3}$.

Vários métodos podem ser utilizados para avaliar a presença de um lado de preferência mastigatória ${ }^{2-11}$. O padrão mastigatório unilateral pode ser conseqüente de sinais e sintomas de disfunção temporomandibular ${ }^{12-15}$, assim como de má oclusão ${ }^{13}$. Entretanto, alguns autores não relacionam o lado de preferência mastigatória com parâmetros oclusais nem com dor muscular, mas sim com o controle nervoso central ${ }^{3}$.

Apesar de existir uma relação anatômica e biomecânica entre a região temporomandibular e crânio-cervical ${ }^{16-22}$, pouco se sabe sobre a influência da postura de cabeça ou postura cervical no desenvolvimento do padrão mastigatório do ser humano. 
Sabe-se que movimentos de extensão, flexão e inclinação de cabeça estão presentes nos humanos durante a mastigação e ocorrem de forma simultânea e rítmica ${ }^{23-25}$.

O objetivo deste estudo foi verificar se existe diferença entre indivíduos com diferentes deformidades dentofaciais (padrão classe II e classe III), em relação à preferência mastigatória referida e inclinação de cabeça durante a mastigação, assim como predomínio intra-grupo de cada um desses aspectos, comparativamente a um grupo controle.

\section{MÉTODO}

Trata-se de um estudo prospectivo, observacional do tipo caso-controle.

Participaram deste estudo, voluntariamente, 25 pacientes entre 15 e 34 anos de idade (idade média de 24,6 anos), 14 homens e 11 mulheres, do Ambulatório de Cirurgia Craniomaxilofacial do Centro Integrado de Estudos das Deformidades da Face (CIEDEF) do Hospital das Clínicas da Faculdade de Medicina de Ribeirão Preto da Universidade de São Paulo (HCFMRP-USP). Esses pacientes formaram o grupo com deformidade (GD). Apresentavam indicação para cirurgia ortognática e foram avaliados apenas no período pré-operatório. Doze pacientes apresentaram o diagnóstico de deformidade dentofacial classe II (GII) (caracterizada por retrognatismo mandibular e/ou por excesso de crescimento maxilar) e 13 pacientes com o diagnóstico de deformidade dentofacial classe III (GIII) (caracterizada por prognatismo mandibular e/ou por deficiência maxilar, tendo a mandíbula mais anteriorizada em relação à maxila).

Também participaram do estudo 10 voluntários saudáveis (4 homens e 6 mulheres), entre 18 e 32 anos (idade média de 23,5 anos), portadores de dentição natural,. Estes indivíduos fizeram parte do grupo controle (GC).

\section{Critérios de inclusão}

Nos grupos com a deformidade dentofacial (GD), foram incluídos indivíduos de ambos os gêneros, com alterações esqueléticas maxilomandibulares observadas no plano sagital classificadas como classe II ou classe III, independentemente de alterações oclusais e esqueléticas no sentido horizontal e/ou vertical (a exceção de mordidas abertas anteriores acentuadas); em uso de aparelho ortodôntico fixo superior e inferior, todos com cirurgia ortognática programada para a correção de tal deformidade.

Para compor o GC foram incluídos indivíduos próximos à faixa etária do GD, de ambos os gêneros, com dentição permanente completa (à exceção dos terceiros molares), sem alterações oclusais, tanto no sentido ântero-posterior, quanto nos sentidos transversais e verticais; em relação molar (Angle) classe I (cúspide mésio-vestibular do primeiro molar superior oclui no sulco vestibular mesial do primeiro molar inferior).

\section{Critérios de exclusão}

Foram excluídos do estudo voluntários portadores de distúrbios neurológicos centrais ou periféricos, que tivessem sofrido traumas e/ou tumores na região de cabeça e de pescoço, ou que tivessem alguma síndrome genética. Também foram excluídos aqueles que usavam próteses dentárias totais ou parciais, ou com ausência de mais de um dente do mesmo lado da arcada dentária, independentemente do espaço interdental.

Do GC foram excluídos os voluntários em uso de aparelho ortodôntico, incluindo aparelho de contenção, com queixas de dor, desconforto ou ruídos na articulação temporomandibular (ATM) e com evidências clínicas de alterações na morfologia da face.

Não foram limitados raça e nível social dos participantes.

Os voluntários da pesquisa foram questionados por uma examinadora sobre a existência de um lado de preferência mastigatória. As possíveis respostas para esse questionamento foram sim ou não, e quando a resposta foi positiva foi solicitada a indicação do lado (direito ou esquerdo). Em sequência, a mastigação habitual de um alimento natural dos mesmos foi filmada pela mesma examinadora. Foi oferecido a cada voluntário um biscoito maizena (Marilanâ, Marília, São Paulo, Brasil) e solicitada à realização da mastigação natural, sem interferência da examinadora. Os mesmos permaneceram sentados, com os pés apoiados no chão, enquanto mastigavam o biscoito. Uma câmera filmadora (handcan-zoom SONY) foi instalada à sua frente sobre um tripé, a distância aproximada de $1 \mathrm{~m}$ e $50 \mathrm{~cm}$ e o voluntário foi orientado a manter o olhar contínuo para a câmara durante a mastigação.

As imagens foram assistidas por outra examinadora que analisou a postura de cabeça de cada voluntário, durante duas mastigações do biscoito.

Para análise das imagens obtidas, primeiramente foi traçada uma horizontal verdadeira entre os lóbulos das orelhas e a partir do momento em que essa linha deixava de ser horizontal era detectada a inclinação de cabeça, que poderia ocorrer tanto para o lado esquerdo quanto para o direito. Para determinação do tempo de inclinação da cabeça, durante o processo de mastigação, um cronômetro digital (Intermec, expect more) foi acionado, na detecção da inclinação da cabeça de um 
determinado lado e interrompido quando do retorno da cabeça a posição neutra ou inclinação para o lado oposto (não mais detectada a inclinação) ou interrompido ainda quando finalizado o processo de mastigação (pela última deglutição do alimento visualizada pela elevação laríngea).

Foi considerado predomínio de inclinação de cabeça quando o voluntário ficou entre 100 a 66,6\% do seu tempo mastigatório (considerando as duas mastigações de cada indivíduo) com a cabeça inclinada.

Todos os participantes foram informados dos objetivos do estudo e assinaram o "Termo de consentimento livre e esclarecido" para participar. O projeto foi aprovado pelo Comitê de Ética em Pesquisa do HCFMRP-USP, processo $\mathrm{n}^{-}$ 2513/2007.

Para a análise das variáveis entre os grupos foi utilizado o teste estatístico Exato de Fisher, e para a análise intra-grupo o teste Binomial, considerandose diferenças significativas $p<0,05$.

\section{RESULTADOS}

Não foi encontrada diferença estatisticamente significativa ( $p>0,05)$ entre os grupos GII, GIII e GC em relação à preferência mastigatória referida (Tabela 1). Entretanto, na análise das variáveis intra-grupo, os grupos com deformidades dentofaciais, Gll e GIII, apresentaram um predomínio significativo $(p<0,05)$ de preferência mastigatória referida.

Não houve diferença estatisticamente significativa $(p>0,05)$ entre os grupos Gll e GIII em relação ao predomínio de inclinação de cabeça durante a mastigação, ou seja, ambos apresentaram predomínio. Entretanto, houve diferença estatisticamente significativa $(p<0,05)$ entre os grupos com deformidade e o GC, evidenciando a inclinação de cabeça durante a mastigação nos dois grupos (GIl e GIII) quando comparados a indivíduos sem deformidades. Esses resultados podem ser visualizados na Tabela 2.

Tabela 1 - Preferência mastigatória referida nos grupos com deformidade dentofacial e grupo controle

\begin{tabular}{cccc}
\hline \multirow{2}{*}{ Variável } & Presença & Ausência & Total \\
\cline { 2 - 4 } & $\boldsymbol{n}$ & $\boldsymbol{n}$ & $\boldsymbol{N}$ \\
\hline GC & 5 & 5 & 10 \\
GII & 10 & 2 & 12 \\
GIII & 11 & 2 & 13 \\
\hline
\end{tabular}

GC $\times$ GII $-p=0.18 ; G C \times$ GIII $-p=0.17$; GII $\times$ GIII- $p=1$; teste Exato de Fisher; N= número de indivíduos; GC= Grupo controle; Gll= grupo classe II; GIII= grupo classe III.

Tabela 2 - Predomínio de inclinação de cabeça durante duas mastigações nos grupos com deformidade dentofacial e grupo controle

\begin{tabular}{cccc}
\hline \multirow{2}{*}{ Variável } & Com Predomínio & Sem Predomínio & Total \\
\cline { 2 - 4 } & $\boldsymbol{n}$ & $\boldsymbol{n}$ & $\boldsymbol{N}$ \\
\hline GC & 8 & 12 & 20 \\
GII & 22 & 2 & 24 \\
GIII & 21 & 5 & 26 \\
\hline
\end{tabular}

GC $\times$ GII - p= 0.0003*; GC x GIII -p=0.0064*; GII x GIII- $p=0.42$; teste Exato de Fisher; N= número de mastigações; $G C=$ Grupo controle; GII= grupo classe II; GIII= grupo classe III.

*Diferença estatisticamente significativa.

$\mathrm{Na}$ análise das variáveis intra-grupo, não houve predomínio significativo $(p>0,05)$ de inclinação de cabeça para os grupos GC e GIII. No entanto, o GII apresentou um predomínio significativo $(p<0,05)$ nesse mesmo aspecto.

\section{DISCUSSÃO}

Os resultados do presente estudo demonstraram a existência de preferência mastigatória referida e predomínio de inclinação de cabeça durante a mastigação nos dois grupos com deformidade 
dentofacial (GII e GIII) quando comparados ao grupo controle (GC).

Várias metodologias são empregadas para avaliar a presença de um lado de preferência mastigatória. Alguns autores aplicaram um questionário perguntando sobre o lado dominante durante a mastigação ${ }^{5,7}$. Métodos objetivos também são utilizados como a eletromiografia ${ }^{4,8,9}$, a observação da mastigação ${ }^{3,10,11}$, a visual spot checking ${ }^{2}$ e a axiografia ${ }^{6}$.

Alguns estudos relacionam a preferência mastigatória com o lado de preferência da mão ${ }^{3,11}$. Nissan et al. ${ }^{3}$ analisaram o lado de preferência das mãos, dos pés, dos olhos, dos ouvidos e da mastigação e observaram que a maioria dos pacientes preferiu o lado direito para todas as funções analisadas. Sendo assim, podemos supor que as atividades funcionais que envolvem lateralidade podem ter um lado de preferência.

Em relação ao lado de preferência ou predomínio mastigatório, outros autores afirmam que podem ocorrer, independente de outras preferências, pois o lado dominante da mão, pé, olho e ouvido são influenciados a nível central, já a mastigação pode ser influenciada por fatores periféricos ${ }^{1,10}$. A escolha por um lado mastigatório específico pode ocorrer através de uma vantagem seletiva de um lado sobre o outro, assim como um maior conforto e eficiência mastigatória no lado preferido.

Outros fatores como alteração na oclusão dentária e disfunção na ATM podem estar relacionados à existência de um lado de preferência mastigatória ${ }^{5,13-15}$. Nossos resultados, na análise das variáveis intra-grupo, mostram uma preferência mastigatória referida em indivíduos com deformidade dentofacial (classe II e III), o que corrobora com a relação oclusal. Acredita-se que com um maior número de sujeitos avaliados poderia se alcançar diferença estatística entre os grupos na avaliação da existência de um lado de preferência mastigatória. A partir disso, sugere-se que um maior número de sujeitos seja estudado nas próximas pesquisas realizadas.

A observação de que o sistema mastigatório e o sistema de regulação postural são anatomicamente e funcionalmente relacionados ${ }^{19}$, leva ao surgimento de diversas hipóteses a respeito da correlação existente entre problemas oclusais e posturais. Michelotti et al. ${ }^{16}$ descreveram evidências da relação entre esses dois sistemas e destacam que tal relação se limita apenas a região crânio-cervical da coluna vertebral, ou seja, não inclui região torácica e lombar.

Uma mudança na posição da cabeça provocada pela contração dos músculos cervicais altera consequentemente, a posição mandibular. Essas mudanças afetam também a oclusão e os músculos mastigatórios e estes, por sua vez, afetam a ATM. De forma inversa, se houver uma má postura mandibular, a posição da cabeça, do pescoço e dos ombros se altera ${ }^{17}$.

De acordo com os resultados obtidos no estudo realizado por Shimazaki et al. ${ }^{18}$ a inclinação lateral do plano oclusal e o desequilíbrio entre os lados direito e esquerdo dos músculos mastigatórios, comum nos indivíduos que possuem um lado de preferência mastigatória, agem contribuindo para o deslocamento da coluna cervical. Contudo, apesar de inúmeros autores afirmarem que existe relação entre a maloclusão e a postura corporal|16,17,20-22, Perinetti et al. ${ }^{26}$ afirmam não existir correlações clínicas importantes entre essas duas variáveis.

Movimentos associados de cabeça podem ocorrer concomitantemente e ritmicamente durante a mastigação. Tais movimentos parecem ser cruciais para a manutenção da função mandibular natural e acontecem devido à ação conjunta dos músculos de cabeça e pescoço e das articulações da coluna vertebral, temporomandibular, e atlanto-occipital ${ }^{25}$.

Dessa forma, quando há abertura e fechamento mandibular a cabeça se move para cima e para baixo, quando a mandíbula se desloca verticalmente durante a mastigação a cabeça se inclina para o lado contrário. Sendo assim, movimentos de extensão, flexão e inclinação de cabeça estão presentes na mastigação ${ }^{16,17}$ e quando o indivíduo desenvolve um lado de preferência mastigatória poderá desenvolver um lado de predomínio de inclinação de cabeça ${ }^{23}$.

O presente estudo mostrou que indivíduos com deformidade dentofacial, ambos os padrões classe II e classe III, apresentaram predomínio de inclinação de cabeça quando comparados aos indivíduos controle, sem apresentar diferença entre eles nesse aspecto. No estudo de $\operatorname{Tay}^{23}$ os indivíduos com má oclusão classe III tenderam a exibir maior inclinação de cabeça quando comparados aos indivíduos classe II. Já nos nossos achados, na análise intra-grupo, houve um predomínio de inclinação de cabeça no Gll. Os sujeitos do nosso estudo apresentaram uma alteração dentária e esquelética, com indicação para correção cirúrgica e não apenas dentária. Esse fato pode justificar a diferença dos nossos resultados.

Outros estudos são necessários para investigar a influência da postura de cabeça ou postura cervical no padrão mastigatório. Segundo $\operatorname{Tay}^{23} \mathrm{o}$ lado de preferência mastigatória geralmente é igual ao lado em que o olho está mais alto, ou seja, o lado oposto da inclinação de cabeça. O autor mostra 
uma correlação entre a preferência mastigatória e o predomínio de inclinação de cabeça.

\section{CONCLUSÃO}

Os resultados do presente estudo demonstraram a existência de preferência mastigatória referida e predomínio de inclinação de cabeça durante a mastigação nos dois grupos com deformidade dentofacial (GII e GIII) quando comparados ao grupo controle (GC). De uma forma geral nossos achados nos permitiram concluir que indivíduos que apresentam deformidade dentofacial estão mais propícios a apresentar um lado de preferência mastigatório e um predomínio de inclinação de cabeça durante a mastigação.

\begin{abstract}
Purpose: the study was conducted in order to investigate the difference among groups with class II and class III dentofacial deformities regarding reported chewing side preference and head inclination during chewing and to determine the intra-group predominance for these features when compared to a control group. Method: twenty-five volunteers aged from 15 to 34 years took part in the study, 12 of them with a diagnosis of class II dentofacial deformity, 13 with class III dentofacial deformity, and 10 control volunteers aged from 18 to 32 years matched for sex and age to the groups with deformity. The subjects were questioned on the presence of a chewing side preference and were recorded during habitual chewing of a cornstarch cookie for analyzing head posture through the images. Results: there was no difference among the groups regarding reported chewing side preference, although the groups with dentofacial deformities had a significant predominance of reported chewing side preference. The groups with dentofacial deformities showed a greater predominance of head inclination during chewing when compared to the control. Conclusion: individuals with dentofacial deformities may show a chewing side preference and changes in head posture during chewing.
\end{abstract}

KEYWORDS: Malocclusion; Posture; Head; Mastication

\section{REFERÊNCIAS}

1. Paphangkorakit J, Thothongkam N, Supanont N. Chewing-side determination of three food textures. J Oral Rehabil. 2006; 33(1): 2-7.

2. Kazazoglu E, Heath MR, Muller F. A simple test for determination of the preferred chewing side. J Oral Rehabil. 1994; 21(6): 723-4.

3. Nissan J, Gross MD, Shifman A, Tzadok L, Assif D. Chewing side preference as a type of hemispheric laterality. J Oral Rehabil. 2004; 31(5): 412-6.

4. Oncins MC, Freire RMAC, Marchesan IQ. Mastigação: análise pela eletromiografia. Seu uso na clínica fonoaudiológica. Distúrbios da Comunicação. 2006; 18(2): 155-65.

5. Diernberger S, Bernhardt O, Schwahn C, Kordass B. Self-reported chewing side preference and its associations with occlusal, temporomandibular and prosthodontic factors: results from the populationbased study of health in Pomerania (SHIP-0). J Oral Rehabil. 2008; 35(8): 613-20.

6. Salioni MA, Pellizoni SE, Guimaraes AS, Juliano $\mathrm{Y}$, Alonso LG. Functional unilateral posterior crossbite effects on mastication movements using axiography. Angle Orthod. 2005; 75(3): 362-7.

7. Strini PJSA, Sousa GC, Júnior R, Strini PJSA, Neto AJF. Alterações biomecânicas em pacientes portadores de Disfunção Temporomandibular antes e após o uso de dispositivos oclusais. Revista Odonto. 2009; 17(33): 42-7.

8. Rahal A, Goffi-Gomez MV. Clinical and electromyographic study of lateral preference in mastication in patients with longstanding peripheral facial paralysis. Int J Orofacial Myology. 2009Nov; 35: 19-32.

9. Ratnasari A, Hasegawa K, Oki K, Kawakami $\mathrm{S}$, Yanagi $\mathrm{Y}$, Asaumi Jl, et al. Manifestation of preferred chewing side for hard food on TMJ disc displacement side. J Oral Rehabil. No prelo 2010.

10. Martinez-Gomis J, Lujan-Climent M, Palau S, Bizar J, Salsench J, Peraire M. Relationship between chewing side preference and handedness and lateral asymmetry of peripheral factors. Arch Oral Biol. 2009; 54(2): 101-7.

11. Nissan J, Berman O, Gross O, Haim B, Chaushu G. The influence of partial implant-supported 
restorations on chewing side preference. $\mathrm{J}$ Oral Rehabil. No prelo 2010.

12. Reinhardt $\mathrm{R}$, Tremel $\mathrm{T}$, Wehrbein $\mathrm{H}$, Reinhardt $W$. The unilateral chewing phenomenon, occlusion and TMD. Cranio. 2006; 24(3): 166-70.

13. Felício CM, Melchior MO, Silva MAMR, Celeghini RMS. Desempenho mastigatório em adultos relacionado com a desordem temporomandibular e com a oclusão. Pró-Fono. 2007; 19(2): 151-8.

14. Casanova-Rosado JF, Medina-Sólis CE Vallejos-Sánchez AA, Casanova-Rosado AJ Hernández-Prado B, Ávila-Burgos L. Prevalence and associated factors for temporomandibular disorders in a group of Mexican adolescents and youth adults. 2006; 10(1): 42-9.

15. Felício CM, Fernandes RSM, Rodrigues da Silva MAM. Investigação da relação entre o tipo mastigatório e o limite de movimento lateral da mandíbula. R Soc Bras Fonoaudiol. 2005; 10(2): 71- 6 .

16. Michelotti A, Manzo P, Farella M, Martina R. Occlusion and posture: is there evidence of correlation? Minerva Stomatol. 1999; 48(11): 525-34.

17. Kibana $Y$, Ishijima T, Hirai T. Occlusal support and head posture. J Oral Rehabil. 2002; 29(1): 58-63.

18. Shimazaki T, Motoyoshi M, Hosoi K, Namura $\mathrm{S}$. The effect of occlusal alteration and masticatory imbalance on the cervical spine. Eur J Orthod. 2003; 25(5): 457-63.
19. Grade R, Caramês J, Pragosa A, Carvalhão J, Sousa S. Postura e disfunção temporomandibular: controvérsias atuais. Rev Port Estomatol Cir Maxilofac. 2008; 49(2): 111-7.

20. Gadotti IC, Berzin F, Biasotto-Gonzalez DA. Preliminary rapport on head posture and muscle activity in subjects with class I and II. J Oral Rehabil. 2005; 32(11): 794-9.

21. D'Attilio M, Caputi S, Epifania E, Festa F, Tecco S. Evaluation of cervical posture of children in skeletal class I, II, and III. Cranio. 2005; 23(3): 218-28.

22. Bergamini M, Pierleoni F, Gizdulich A, Bergamini C. Dental occlusion and body posture: a surface EMG study. Cranio. 2008; 26(1): 25-32.

23. Tay DKL. Physiognomy in the classification of individuals with a lateral preference in mastication. $J$ Orofac Pain. 1994; 8(1): 61-72.

24. Eriksson PO, Zafar H, Nordh E. Concomitant mandibular and head-neck movements during jaw opening-closing in man. J Oral Rehabil. 1998; 25(11): 859-70.

25. Igarashi N, Yamamura K, Yamada $\mathrm{Y}$, Kohno $\mathrm{S}$. Head movements and neck muscle activities associated with the jaw movement during mastication in the rabbit authors. Brain Res. 2000; 871(1): 151-5.

26. Perinetti G, Contardo L, Biasati AS, Perdoni L, Castaldo A. Dental malocclusion and body posture in young subjects: a multiple regression study. Clinics. 2010; 65(7): 689-95.

DOI: $10.1590 / S 1516-18462010005000133$

RECEBIDO EM: 26/07/2010

ACEITO EM: 20/08/2010

Endereço para correspondência:

Marcela Ralin de Carvalho Deda

Rua Arnaldo Victaliano,700, apt. 611, Jardim Palma Travassos

Ribeirão Preto - SP

CEP: 14091-220

E-mail: marceladeda@yahoo.com.br 